\title{
Huge Capillary Haemangioma of the Nasal Fossae
}

\author{
Khdim M*, Choukry K, Chaouki A, Abada R, Rouadi S, Roubal M and Mahtar M \\ ENT Department and Head and Neck Surgery, Ibn Rochd Teaching Hospital, Casablanca, Morocco
}

${ }^{*}$ Corresponding author: Khdim M, ENT Department and Head and Neck Surgery, Ibn Rochd Teaching Hospital, Casablanca, Morocco, Tel: 0615372251, E-mail: Khdim02mouna@gmail.com

Citation: Khdim M, Choukry K, Chaouki A, Abada R, Rouadi S, et al. (2019) Huge Capillary

Haemangioma of the Nasal Fossae. J Case Rep Stud 7(2): 205. doi: 10.15744/2348-9820.7.205

Received Date: November 22, 2018 Accepted Date: April 25, 2019 Published Date: April 27, 2019

\begin{abstract}
Introduction: Hemangiomas are frequently observed lesions in the head and neck region, those of the nasal cavity and sinuses are very rare. They are divided into two histological types, capillary and cavernous ones.

Materials and Methods: We report a case of a 60-years-old female with two years' history of recurrent episodes of epistaxis associated with nasal obstruction. The clinical examination had found swelling of the right hemi face and infiltrations of both orbits causing a bilateral eyelid closure.

Results: a facial MRI showed a large mass of the right nasal cavity with hyposignal on T1, hypersignal on T2 and intense enhancement with gadolinium, extending to the cavum with loco regional invasion of ipsilateral maxillary sinus, optic chiasma, optic nerves with and frontal lobe. Rhinocavoscopy was performed under local anesthesia, there was a complete obstruction of the right nasal cavity with a vascular appearing mass, displacing the nasal septum on the controlateral side, making exploration of the left nasal cavity difficult. The rhinocavoscopy was followed by a biopsy of the mass causing a significant bleeding that was controlled with nasal packaging. Blood transfusion was not needed. The anatomo pathological examination of the specimen revealed a capillary hemangioma of the nasal cavity.
\end{abstract}

Conclusion: Hemangiomas of the nasal and paranasal cavities are benign tumors, rarely reported in the literature. The treatment of these lesions is difficult and it is not well codified, especially when they reach a large volume with a loco regional extension.

Keywords: Huge; Capillary Hemangioma; Epistaxis; Biopsy; Radiotherapy

\section{Introduction}

Hemangiomas are frequent lesions of the head and neck region, those in the nasal cavity and sinuses are very rare, only 65 cases have been reported in the literature [1,2]. Their etiology is unknown. There appears to be a hormonal component as these lesions occur commonly in pregnancy and in patient on oral contraceptive pills [3]. There are also reports of the development of the pathology following local trauma [4,5]. There are two histological types, capillary and cavernous ones. The capillary hemangioma is more common in pediatric population affecting the nasal septum and regress after few years [1]. We report a case of a female patient with a capillary hemangioma of the nasal cavity.

\section{Clinical Case}

A 60-years-old female without any history of nasal packaging, or facial trauma, admitted to our ENT department with two years' history of recurrent episodes of epistaxis associated with nasal obstruction. The clinical examination had found swelling of the right hemi face and infiltrations of both orbits causing a bilateral eyelid closure, explaining the difficulty of performing an oculomotricity examination and evaluating the optic nerve function (Figure 1). However, the patient reported progressive vision deterioration. The rest of neurological examination had not found any abnormalities. The computed tomography (CT) scan was out of working order in the hospital, so we performed a facial MRI that showed a large mass of the right nasal cavity with hyposignal on T1, hypersignal on T2 and intense enhancement with gadolinium, extending to the cavum with loco regionalinvasion of ipsilateral maxillary sinus, optic chiasma and optic nerves with invasion of the frontal lobe (Figure 2 and 3 ). Rhinocavoscopy was performed under local anesthesia, there was a complete obstruction of the right nasal cavity with a vascular appearing mass, displacing the nasal septum on the controlateral side, making exploration of the left nasal cavity difficult.

The rhinocavoscopy was followed by a biopsy of the mass causing a significant bleeding that was controlled with nasal packaging. Blood transfusion was not needed. The anatomo pathological examination of the specimen revealed a capillary hemangioma of the nasal cavity. 


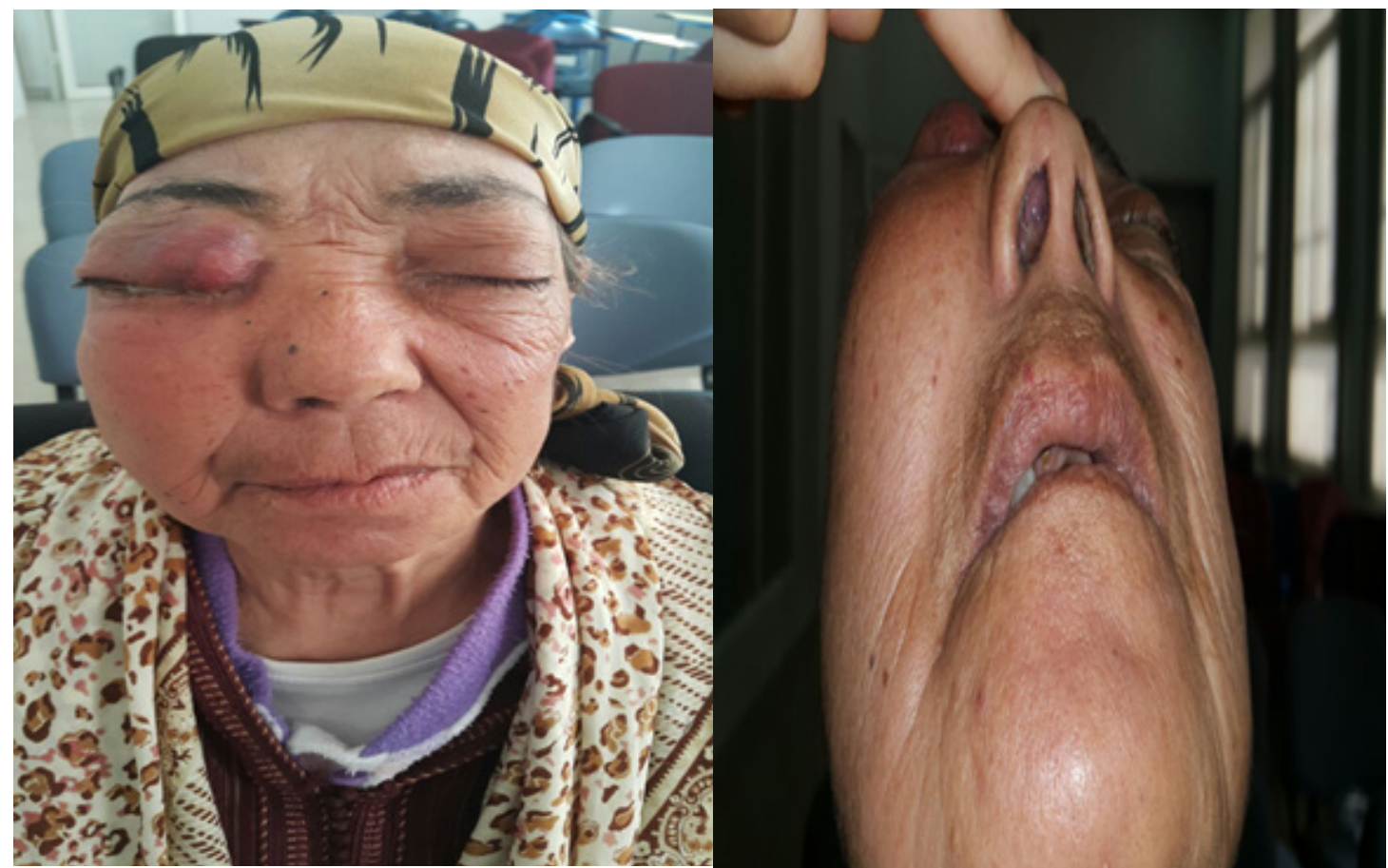

Figure 1: Swelling of the Right Hemi Face and Infiltrations of both Orbits Causing a Bilateral Eyelid Closure

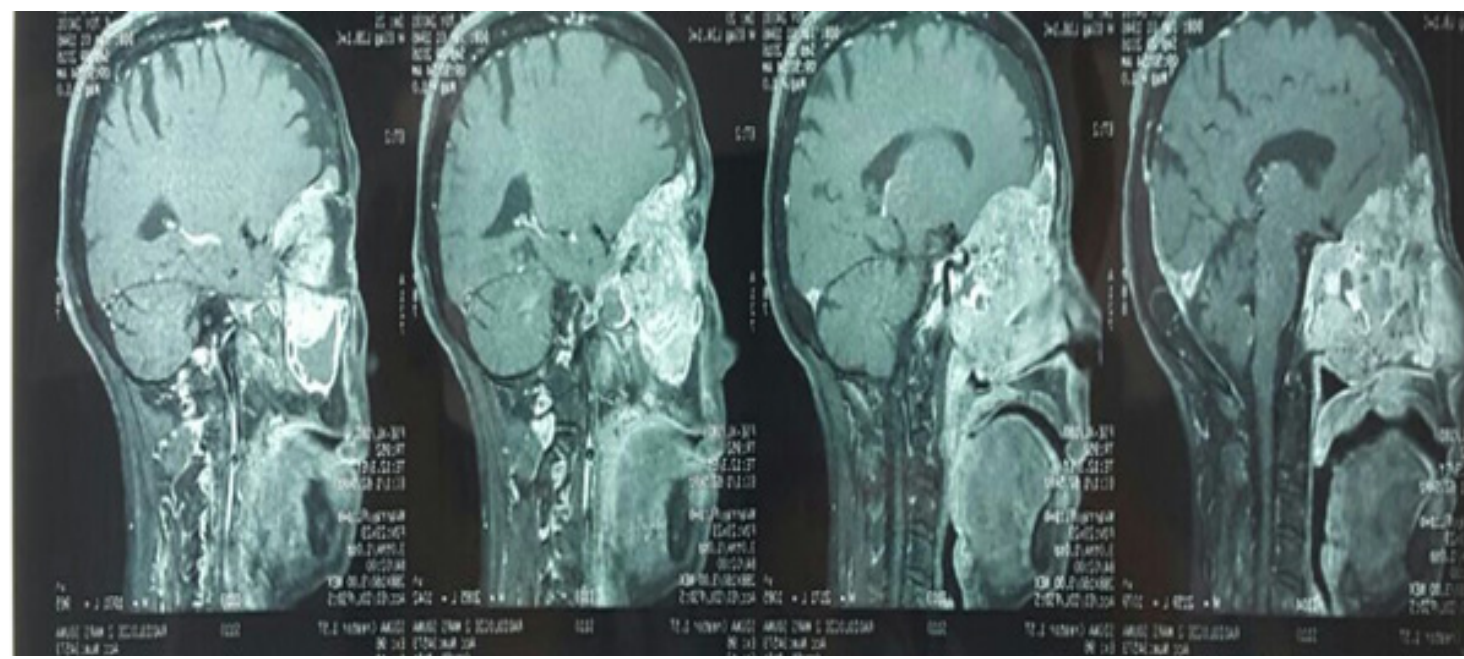

Figure 2: MRI of the Face Showing a Capillary Haemangioma of the Right Nasal Fossa

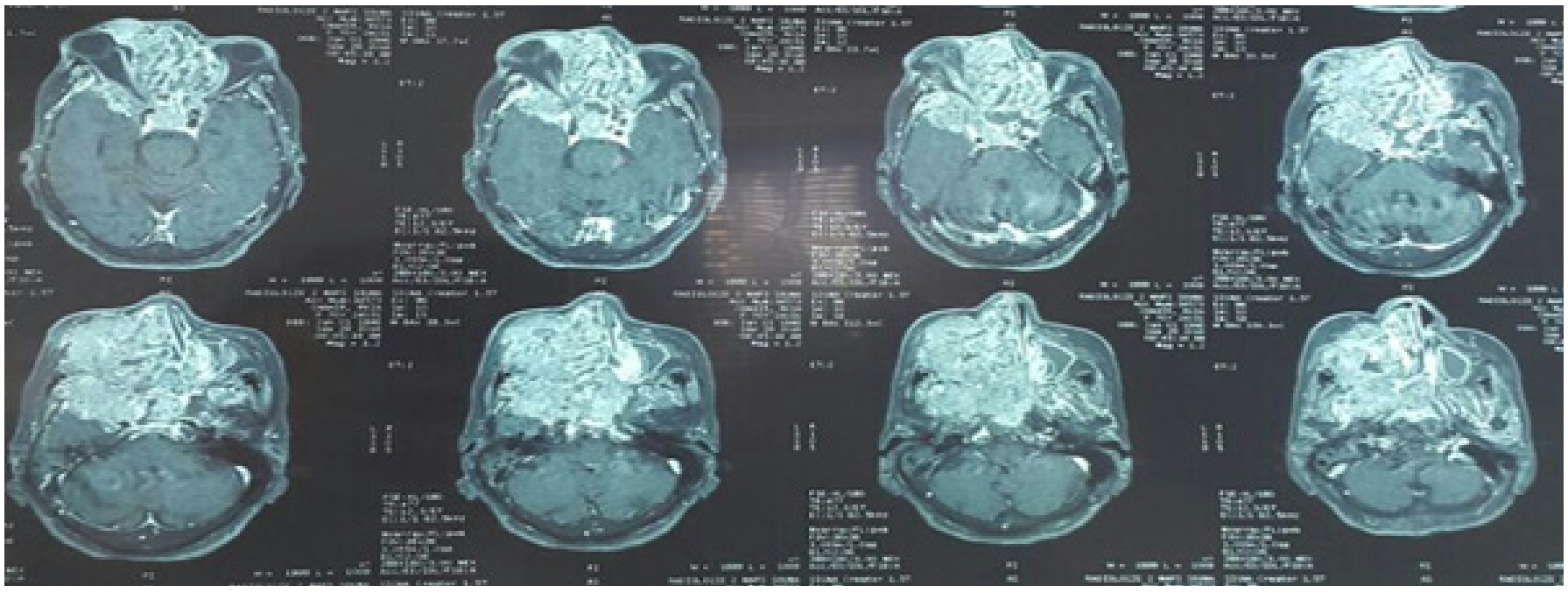

Figure 3: Tissue mass enhanced with Gadolinium Injection, with extension to Neighboring Organs 
After the confirmation of the diagnosis, the multidisciplinary meeting's treatment decision was radiotherapy because of the extension of the lesions. Two months later, the patient passed away due to the tumor extension.

\section{Discussion}

Hemangiomas of the nasal and paranasal cavities are benign tumors, rarely reported in the literature [6]. Their etiology is unknown. There appears to be a hormonal component as these lesions occur commonly in pregnancy and in patient on oral contraceptive pills [3]. There are also reports of the development of the pathology following local trauma [4,5].

Hemangiomas are divided into two histological types, capillary and cavernous, depending on the size of the dominant vessels on microscopic examination [2]. They can arise from skin, mucous membranes and deep structures such as bone or glands. The evolution of this pseudo tumor is slow with a destructive tendency in relation to its compressive effect. In the nasal cavity, about $80 \%$ of the hemangiomas originate from the anterior part of the nasal septum (Kiesselbach triangle); the capillary hemangiomas are generally well circumscribed tumors [4,5]. Capillary hemangiomas often occur early in life, whereas cavernous hemangiomas mainly affect adults between the 4th and 5th decades, supporting the hypothesis that they are acquired lesions. The most common symptom is unilateral epistaxis with nasal obstruction similar to our case report.

The appearance in endoscopy is non-specific; it shows a red purplish mass, rounded on a normal nasal mucosa [2]. On MRI, in T2 sequences, the mass is hypersignal, hemosiderin deposits at the periphery of the mass are hyposignal. The presence of hypo intense foci is related to phleboliths, more characteristic of cavernous hemangiomas. MRI allows a better evaluation of the tumoral extension to the deep spaces of the face. It also makes it possible to distinguish between sinus invasion and retentions. The treatment of nasal hemangiomas is extensive excision with tumor, mucosa and underlying perichondrium $[4,5]$. Unfortunately, recurrence rate after excision can be as high as $15 \%$ [7]. For nasal hemangiomas without any other extension, an endonasal endoscopic approach is preferred. For large and extensive tumors, preoperative embolization should be done. Although radiotherapy is a good treatment choice for hemangiomas, long-term side effects, such as malignancy, region growth impairment, and scarring, makes it an unfavorable treatment modality. Therefore, radiotherapy is only used for unresectable lesions [8-10].

In our case report, our patient had radiotherapy without any surgical excision. Other therapeutic methods can be performed for extensive tumors such as sclerotherapy, cryotherapy and YAG laser [11].

\section{Conclusion}

Hemangiomas of the nasal and paranasal cavities are benign tumors, rarely reported in the literature. The treatment of these lesions is difficult and it is not well codified, especially when they reach a large volume with loco regional extension.

\section{References}

1. Gourin CG, Millay DJ (2000) Pathology forum: quiz case 3. Diagnosis: cavernous hemangioma of the nasal bones. Arch Otolaryngol Head Neck Surg 126: 902-7.

2. Ash JE, Old JW (1950) Hemangiomas of the nasal septum. Trans Am Acad Ophtalmol Otolaryngol 54: 350-6.

3. Dettelbach M, Weissman J, Singh J, Eibling D (1995) Bleeding Polyp" of the osseous nasal septum: a rarely seen lesion. Am J Otolaryngol 16: 341-6.

4. Fahmy F, Back G, Smith C, Hosni A (2001) Osseous haemangioma of inferior turbinate. J Laryngol Otol 115: 417-8.

5. Zainine R, Marrakchi J, Akkari S, Chahad H, Mediouni A (2012) The hemangiomas of cornets: an entity not to forget! (Les hémangiomes des cornets: une entité à ne pas oublier!. Annales françaises d'Oto-rhino-laryngologie et de Pathologie Cervico-faciale). French Ann Otorhinolaryngol Cervico-facial Pathol 129 : A98.

6. Webb CJ, Porter G, Spencer MG, Sissons GR (2000) Cavernous haemangioma of the nasal bones: an alternative management option. J Laryngol Otol 114: 287-9.

7. Mills SE, Cooper PH, Fechner RE (1980) Lobular capillary hemangioma: the underlying lesion of pyogenic granuloma. A study of 73 cases from the oral and nasal mucous membranes. Am J Surg Pathol 4: 470-9.

8. Takeda K, Takenaka Y, Hashimoto M (2010) Intraosseous hemangioma of the inferior turbinate. Case Rep Med 2010: 409429.

9. Iwata N, Hattori K, Nakagawa T, Tsujimura T (2002) Hemangioma of the nasal cavity: a clinicopathologic study. Auris Nasus Larynx 29: 335-9.

10. Çaylakli F, Çağici AC, Hürcan C, Bal N, Kizilkiliç O, (2008) Cavernous hemangioma of the middle turbinate: a case report. Ear Nose Throat J 87: 391-3.

11. Webb CJ, Porter G, Spencer M, Sissons G (2000) Cavernous haemangioma of the nasal bones: an alternative management option. J Laryngol Otol 114: 287-9. 


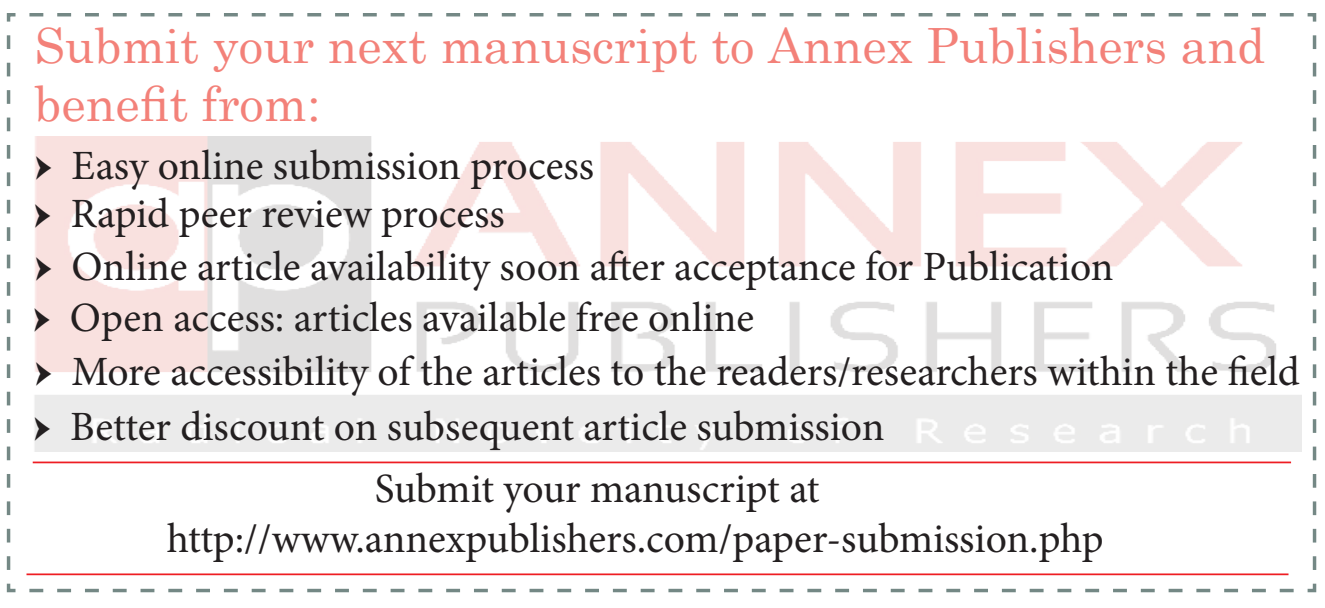

